\title{
Leakage of long-period oscillations from the chromosphere to the corona
}

\author{
D. Yuan ${ }^{1}$, V. M. Nakariakov ${ }^{1,2}$, N. Chorley ${ }^{1}$, and C. Foullon ${ }^{1}$ \\ ${ }^{1}$ Centre for Fusion, Space and Astrophysics, Department of Physics, University of Warwick, Coventry CV4 7AL, UK \\ e-mail: Ding.Yuan@warwick .ac.uk \\ 2 Central Astronomical Observatory at Pulkovo of the Russian Academy of Sciences, 196140 St Petersburg, Russia
}

Received 21 March 2011 / Accepted 7 August 2011

\begin{abstract}
Long-period oscillations in a coronal diffuse structure are detected with the Transition Region And Coronal Explorer (TRACE). The EUV images of the NOAA active region 8253 are available in $171 \AA$ and $195 \AA$ bandpasses from 30 June to 4 July 1998 . The average intensity variation is found to be connected with the CCD temperature, which varies with the orbital motion of the spacecraft. Hence, oscillations with the orbital period and its higher harmonics appear as artifacts in the light curves. After the exclusion of the orbital effects, we identified several long-period oscillations in the diffuse fan-like structure of the active region. Similar periodicities were detected in the radio emission from the chromospheric part of that active region, observed with the ground-based Nobeyama Radioheliograph (NoRH) in the $17 \mathrm{GHz}$ channel. It was found that $0.221,0.312$ and $0.573 \mathrm{mHz}$ oscillations were present in both EUV emission lines in the corona and the radio signal from the sunspot in the chromosphere, just beneath the active region. From the frequency values, the 1 st and 3rd detected oscillations could be associated with the $l=2, n=-3$ or $l=3, n=-5$ and $l=1$ gravity-driven solar interior modes, respectively. The appearance of these oscillations in the coronal part of the active region can be connected with the wave leakage or the evanescence of chromospheric oscillations.
\end{abstract}

Key words. Sun: oscillations - Sun: corona - Sun: UV radiation - Sun: atmosphere - sunspots - instrumentation: miscellaneous

\section{Introduction}

Propagating intensity disturbances in Extreme UltraViolet (EUV) bandpasses were first observed in the polar plumes (Deforest \& Gurman 1998) and over the strands of large coronal loops (Berghmans \& Clette 1999) with SOHO/EIT. This stimulated a number of follow-up studies, mainly in the $171 \AA$ and $195 \AA$ bandpasses of TRACE (e.g. De Moortel et al. 2000, 2002a,b; King et al. 2003). The intensity disturbances are observed to propagate at the speeds lower than the local sound speed due to projection effects. The typical periods of these disturbances were found to cluster near 3 and $5 \mathrm{~min}$ in the power spectrum (De Moortel et al. 2002a). Hence, they are likely to be associated with the chromospheric 3-min oscillations and the photospheric 5-min oscillations, respectively. The 3-min oscillations were found to leak from sunspot umbra to the corona (Shibasaki 2001; Sych et al. 2009). The 5-min oscillations, above the acoustic cut-off period, can be channelled to the corona by the inclined magnetic field lines (e.g above the plage region, see De Pontieu et al. 2005). The coronal propagating disturbances are usually interpreted as propagating slow magnetoacoustic waves (Nakariakov et al. 2000; Verwichte et al. 2010).

Longer period oscillations are detected in sunspot chromosphere and transition region (Nagovitsyna \& Nagovitsyn 2001, 2002). The oscillations of tens up to hundreds of minutes were observed as modulations of the radio emission (Gelfreikh et al. 2006). The line-of-sight velocity oscillations with periods ranging from 60 to $80 \mathrm{~min}$ were found in the analysis of the Doppler shift of spectral lines in sunspots (Efremov et al. 2009). Long-period oscillations (16-88 $\mathrm{min}$ ) of the radio emission intensity over sunspots were recently studied with the Nobeyama Radioheliograph (NoRH) (Chorley et al. 2010, 2011).

Theoretical studies argue that these long-period (low frequency) oscillations are well below the cut-off frequency (e.g. Bel \& Leroy 1977), thus cannot appear in the corona. However, in the corona, long-period oscillations have been detected in the EUV emission with multiple instruments and in different bandpasses. For example, propagating waves with long periods (10-15 min) were observed in coronal loops with EIT (Berghmans \& Clette 1999), TRACE (McIntosh et al. 2008) and STEREO/EUVI (Marsh et al. 2009). Wang et al. (2009) reported two significant periodicities of 12 and $25 \mathrm{~min}$ in the intensity and Doppler shift variations over a coronal diffuse structure with Hinode/EIS. Prominence oscillations with periods ranging from 20 min up to several hours were reported as well (Foullon et al. 2004, 2009; Tripathi et al. 2009; Hershaw et al. 2011).

Likewise, long-period oscillations have been observed in the upper corona and the solar wind. In particular, Ofman et al. (2000) detected an oscillation of the polarised white-light brightness with a $30 \mathrm{~min}$ period at $1.9 R_{\odot}$ in a polar coronal hole. Similar periodicities in the low-energy charged particle flux were found to be modulated by the variation of the interplanetary magnetic field, which may be of solar origin (Thomson et al. 1995). Moreover, Kepko \& Spence (2003) established a high correlation of solar wind density variations and the ultra low frequency (ULF) oscillations of the Earth's magnetosphere, with the power spectrum peaks being located at $0.1,0.2$ and $0.6 \mathrm{mHz}$.

Thus, there appear questions as to whether the long-period oscillatory phenomena, observed in different parts of the solar atmosphere, are connected with one another, and how this connectivity is carried out. For example, for 3-min chromospheric 


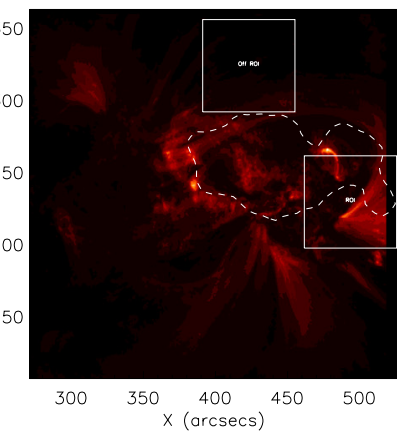

(a)

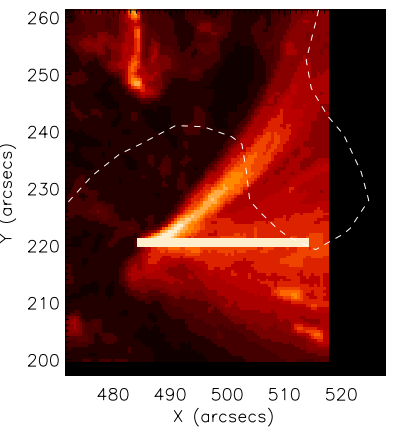

(b)

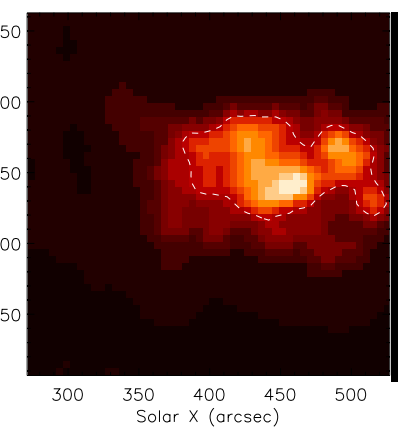

(c)

Fig. 1. a) The field of view over AR8253 taken at 1998-07-01 01:01 UT in TRACE 171 $\AA$ bandpass, the region of interest (ROI) in the white box includes the fan-like structure and is re-sized to the left, an off-ROI region including the weakest intensity area is marked to the top. b) The region of interest $(128 \times 128$ pixels $)$ showing the fan-like structure; a slit of the macro-pixels $(3 \times 3)$ is selected along the fan. c) The intensity map of $17 \mathrm{GHz}$ radio emission over AR8253 at 1998-07-01 01:01 UT. The contour in dashed line marked the sunspot area.

oscillations in sunspots, the possibility of the acoustic wave leakage into the corona has recently been demonstrated numerically (Botha et al. 2011), but for longer periods a similar mechanism has not been developed. Also, a related question is whether the atmospheric long-period oscillations are anyhow connected with the long-period gravity-driven oscillations of the solar interior. Indeed, the solar $g$-mode periods are estimated in the range of 16 min to $28 \mathrm{~h}$ (e.g. Appourchaux et al. 2010), these are similar to the periods of the atmospheric oscillations. The aim of this paper is to contribute to our understanding of this connectivity.

Here, we analyse long-duration observations of active region AR8253 in the $171 \AA$ and $195 \AA$ bandpasses of TRACE, for five days. We aim to fully take advantage of the long dataset and search for the long-period oscillations and its originality in combination with NoRH $17 \mathrm{GHz}$ radio data. The dataset and its pre-processing are described in Sect. 2; the orbital effects are discussed in Sect. 3; the power spectrum analysis and the elimination of the orbital artifacts are presented in Sect. 4, and final conclusions are given in Sect. 5.

\section{Dataset}

\subsection{Observations}

TRACE targeted AR8253 almost continuously for about 5 days, from 30 Jun. to 4 Jul. 1998. The images were taken with the half field-of-view (FOV), $512 \times 512$ pixels, at the full resolution of $0.5 \mathrm{arcsec} /$ pixel in $171 \AA$ and $195 \AA$ bandpasses. The typical cadence time was either $\sim 41$ or $\sim 30 \mathrm{~s}$. The imaging interval between $171 \AA$ and $195 \AA$ was normally 11 s. The data contained several gaps, lasting from $\sim 20 \mathrm{~min}$ to $\sim 4 \mathrm{~h}$, but the total observation time covered about $\sim 70 \%$ of the time span in both EUV bandpasses. Radio observations at $17 \mathrm{GHz}$ were provided by NoRH. It operates daily from $22: 45$ to $6: 30$ UT of the next day, providing data at $10 \mathrm{~s}$ cadence with spatial resolution of $10 \mathrm{arcsec} / \mathrm{pixel}$.

\subsection{Pre-processing}

The TRACE datacubes were prepared with the standard routine TRACE_PREP in Solar Software with standard pre-processing keywords. The EUV images were calibrated with white-light pointing; the spikes and streaks from radiation belts and cosmic rays, plus the readout noise, were removed; the flux intensities were normalised to the exposure time. The image coordinates were co-aligned at the origin of the solar disk in Heliocentric-Cartesian Coordinates to correct the spacecraft retargeting. The fan-like structure (Fig. 1a) was tracked with the co-moving frame following the solar surface differential rotation. The spacecraft pointing drifts (Handy et al. 1999), plus the uncertainties of solar rotational model, were minimised by cross-correlation offsets. Two co-aligned sub-datasets $(128 \times$ 128 pixels) were taken for further analysis (Fig. 1b).

The NoRH $17 \mathrm{GHz}$ radio data was synthesised with the Koshix algorithm. The sunspot was located in the full-disk image and followed with a solar co-rotating box of size $(36 \times 36$ pixels $)$ (see details in Chorley et al. 2010). The FOV was chosen to coincide with the EUV observations. The position of the region of the microwave emission intensity is contoured in dashed line in the EUV image in Fig. 1c. The strong gyro-resonant emission is situated in the sunspot chromosphere. Above it, the fan-like diffuse structure in the corona is an magnetic field extension from the sunspot (Fig. 1a).

\subsection{Co-alignment and dejittering}

As the TRACE observation spans over $100 \mathrm{~h}$, the images had to be co-aligned and de-jittered. Four images were selected for each day as the reference for cross-correlation and were coaligned by overlapping the footpoint within an accuracy of less than 1.5 pixels. The rest of the images were smoothed with boxcar of $10 \times 10$ pixels, then aligned by the offset calculated by cross-correlating to the corresponding reference image. The offsets were treated as time series and smoothed by running averaging to suppress the noise. This approach is more efficient than any smoothing in the images. However it becomes very unstable to the outliers introduced by disruption(s) to the image topology.

There are three types of outliers (Gounder et al. 2007) in the offsets: 1) additive outliers (AO) are single exotic values, normally due to CCD saturation bleeds that ruin part(s) of an image. AOs are easily detected by thresholding and replaced with neighbouring values; 2) innovational outliers (IO) appear as an abrupt step of the average, during which part of the region of interest is out of the FOV; IOs are corrected by restoring the mean; 3 ) transitory change outliers (TCO) are spikes in the time series that disappear gradually. A flare or a bright point normally induces TCO. TCOs are replaced by linearly interpolated values between the points before and after the event (about $0.5-1 \mathrm{~h}$ ). 


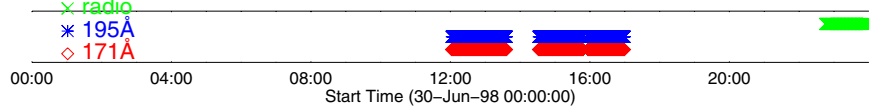
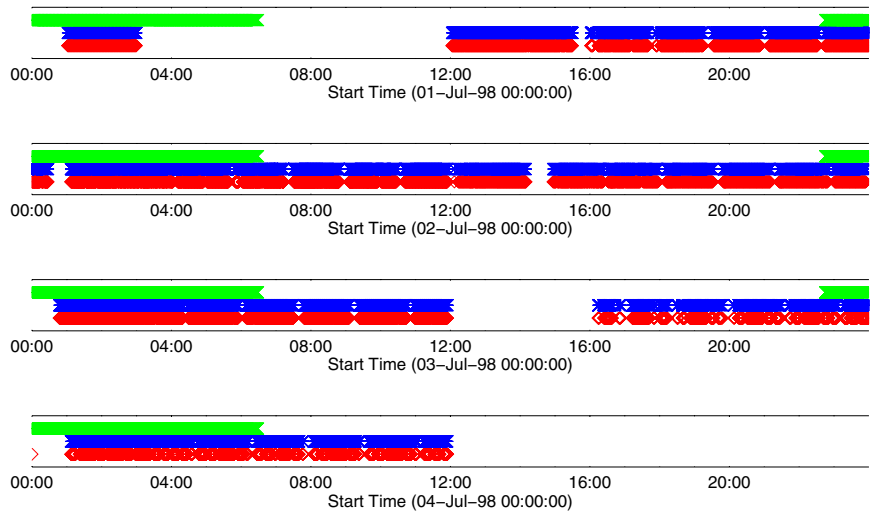

Fig. 2. Observational coverage over AR8253 by TRACE $171 \AA$ (red) and $195 \AA$ (blue), and NoRH $17 \mathrm{GHz}$ (green) in the analysed time interval. Small gaps in EUV observations of order $100 \mathrm{~s}$ are due to channel switching, and large ones are due to routine observations on the solar limb (including polar regions), re-pointing or to avoid radiation belts.

The global accuracy of the co-alignment over 5 days is less than 1.5 pixels, the local relative accuracies (over several hours) are normally as good as 0.5 pixel. The appearances of TCOs deteriorate the co-alignment accuracy to $\sim 2$ or 3 pixels for about $0.5-1 \mathrm{~h}$, these parts are deliberately avoided in later analysis.

\subsection{Spectral analysis}

In the selected time interval, the active region AR8253 was observed for the time sufficiently long for the detection of longperiod oscillations, with least disruptions. In the selection of a suitable time interval, we opted to minimise the presence of large data gaps, as a time series containing them lacks a significant portion of information, the power spectrum may be totally erroneous irrespective of the spectral analysis technique. The analysed series of the EUV images were not uniformly sampled, and the data has to be re-sampled into uniform time grid by interpolation. Even though, as the data contained some gaps, its power spectrum was obtained with the Lomb-Scargle periodogram technique (Scargle 1982; Horne \& Baliunas 1986). This technique is specifically applicable to the analysis of unevenly sampled data. It overcomes the difficulty posed by unevenness, reduces side-lobe effect and can focus on a certain range of frequencies.

De-trending is applied to the time series by removing the running average before spectral analysis. This process removes ultra-long period trend, it is normally comparable to the range of the time series and contains the large portion of the total power. De-trending relatively suppress the low-frequency bandpass and levitate the high-frequency part. We remove 30 min running average in the time series in studying the orbital effect (Fig. 3), a different averaging time only results in changes in relative power of the peaks. In obtaining Fig. 4, we subtracted 60-min running average from the time series.

In order to distinguish the peaks out of orbital artifacts (see Sect. 3), we applied temporal filtering to the time series. The orbital harmonics are removed by subtracting sinusoidal functions from the time series iteratively with date-compensated discrete
Fourier transform (DCDFT),

$\hat{y}(t)=y(t)-\left[d_{0}+d_{1} \sin (2 \pi f t)+d_{2} \cos (2 \pi f t)\right]$

where $f$ is a orbital harmonic frequency, the coefficients $d_{0}, d_{1}$, $d_{2}$ are estimated according to Ferraz-Mello (1981). Spectral filtering was also attempted, an abrupt window would bring in edge effects to the spectrum, while a smooth one may not completely remove the peak (see Inglis \& Nakariakov 2009). DCDFT can be done without tackling the spectral domain, it calculates the accurate magnitude of the frequencies, but sometimes residual peaks may appear around the removed component (see Fig. 4e, $\mathrm{f}$ and discussions in Sect. 5).

Referring to the significance level of the detected peaks, no scheme is well accepted by the community, as the distribution of random noise is unknown, a certain assumption of noise distribution has to be made. Moreover, the processing of the data, e.g. de-trending, filtering, destroys the independence of the measurements and coherence of the time series (Hernandez 1999), while these steps are normally unavoidable.

We estimated the false alarm probability (FAP) at 0.01 to the spectrum, assuming Gaussian noise distribution based on Horne \& Baliunas (1986). FAP is the probability to reject a detected peak, it only applies to the highest peak in the spectrum, thus, we subtract the highest peak from the time series using Eq. (1) and repeat this process to the rest of the peaks until all whole spectrum is below the significant level. All peaks quoted in the following text are lower than 0.01 significance level by default, unless specified such as the bracketed numbers in Table 1, they are illustrated in iteration order in Fig. 5 (171 ̊), Fig. 6 (195 ̊) and Fig. 7 (17 GHz radio).

We also perform Fisher's randomisation test (Linnell Nemec $\&$ Nemec 1985; O'Shea et al. 2001) to all the detected peaks in the power spectrum. This is an unbiased distribution-free test, that estimates the significance level ( $p$-value) of the peaks. Consider a time series, $y_{i}=y\left(t_{i}\right)$ and its periodogram, $F_{0}(\omega)$. By randomly permuting pairs of data points, $y_{\delta_{k}} \leftrightarrow y_{\delta_{j}}$, the time series is rearranged, while the noise remains intact. If there is a significant periodic component in the data, say $T_{n}=2 \pi / \omega_{n}$, and $\left|t_{\delta_{i}}-t_{\delta_{j}}\right|=T_{n}$, there is a certain probability that we detect a higher peak at $\omega_{n}$. Repeat this process $M$ times, if a higher peak is recorded $K$ times at $\omega_{n}$, the Fisher's randomisation estimates the false alarm probability as $p=K / M$ ( $p$-value is alternative to $F A P$ in Horne \& Baliunas 1986, test), the standard error is $(p(1-p) / M)^{0.5}$. We run 1000 permutations in our studies, the maximum uncertainty is $\sigma(p)=0.5 M^{-0.5}=0.016$. If $p=0.0$ is obtained, we quote as $p<0.01$ by considering the error bar. It is done by iteratively removing the highest peak in the spectrum as described by Eq. (1). We use the time series analysis package PERIOD developed by the Starlink Project (Dhillon et al. 2001). All results are included in Table 1.

\section{Orbital effect}

TRACE orbits in a polar sun-synchronous orbit, roughly following the solar terminator (twilight zone). The orbital period is $\sim 96 \mathrm{~min}(0.17 \mathrm{mHz})$. Because of the orbital motion, the EUV flux incident on the detector changes periodically, thus the CCD temperature is modulated and varies with the orbital period (see Fig. 3, top row). The changes in temperature introduce variability to the pedestal value of the observed intensity and the readout noise (Aschwanden et al. 2000). It should be accounted for when studying long-period intensity oscillations.

The analysis of the time variation of pixel count averaged over the ROI in $171 \AA$ data (see Fig. 1a) in 1998-07-03 

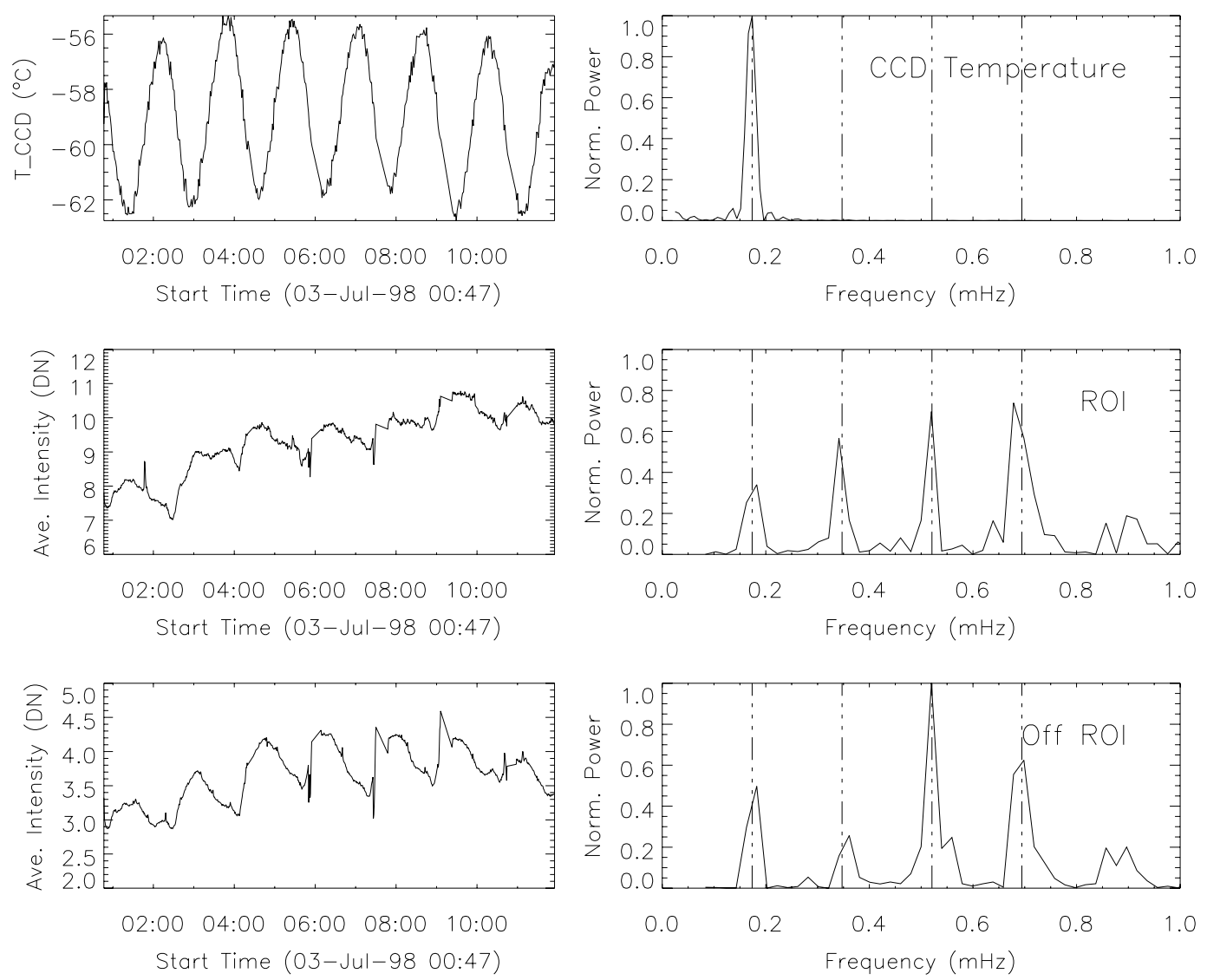

Fig. 3. Left column: time series (1998-07-03 00:47-12:00 UT). Right column: the corresponding normalised power spectra, a 30-min running average is removed from the intensity time series beforehand, the harmonics of the orbital periods are marked with the vertical dash-dot-dot lines, corresponding to the periods 96, 48, 32, $24 \mathrm{~min}$. From top to bottom row: TRACE CCD temperature variation $\left({ }^{\circ} \mathrm{C}\right)$, the average intensity $(\mathrm{DN})$ of the ROI, and off ROI.

00:47-12:00 UT (Fig. 3, middle row) revealed pronounced peaks at the frequency corresponding to the orbital period $0.17 \mathrm{mHz}$ (96 min) and its higher harmonics $0.35 \mathrm{mHz}$ (48 min), $0.52 \mathrm{mHz}$ (32 $\mathrm{min}), 0.69 \mathrm{mHz}$ (24 min). The same periodicities were detected in a rather quiet region (Fig. 3, bottom row), chosen off the ROI region (Fig. 1a). The same result are found in both $171 \AA$ and $195 \AA$ data. The presence of the higher harmonics of the orbital period is connected with nonlinear dependence of the image intensity to the CCD temperature (Fig. 3, top row).

By assuming the CCD temperature $T=T_{0}+\delta T \cos \left(\frac{2 \pi t}{P}+\phi\right)$, we expand the average intensity $F(T)$ of the images in to Taylor series and re-arrange it by trigonometric operations:

$$
\begin{aligned}
F(T) & =F\left(T_{0}\right)+\frac{\mathrm{d} F}{\mathrm{~d} T} \delta T+\frac{\mathrm{d}^{2} F}{2 ! \mathrm{d} T^{2}}(\delta T)^{2}+\ldots \\
& =a_{0}+a_{1} \cos \left(\frac{2 \pi t}{P}+\phi\right)+a_{2} \cos ^{2}\left(\frac{2 \pi t}{P}+\phi\right)+\ldots \\
& =b_{0}+b_{1} \cos \left(\frac{2 \pi t}{P}+\phi_{1}\right)+b_{2} \cos \left(\frac{2 \pi t}{P / 2}+\phi_{2}\right)+\ldots
\end{aligned}
$$

Here the harmonics periods affect the image intensities, as the telescope orbits around the Earth, it is due to the non-linearity of the orbital environment, the detection efficiency, the telescope system. The orbital effect applies to other space missions as well, e.g. Yohkoh, Hinode and CORONAS-Photon. It varies between instruments and has to be studied systematically to avoid misleading results.

\section{Results}

We selected a time series (1998-07-03 00:47-09:00 UT) of a derotated macro-pixel $(3 \times 3$ pixels $)$ situated along the mid-way of the slit (Fig. 1b) in the image in $171 \AA$. A time interval affected by a C1.3 GOES-class flare (11:19-11:33 UT) was cropped. There are several images corrupted by CCD saturation bleeding between 09:00-10:00 UT, and we simply crop this part. We attempted removing the bad images manually, and included one more hour to the time series, but it resulted in tiny difference compared to Fig. 4. The power spectrum of the signal is shown in Fig. 4a. It contains a mixture of peaks, including the orbital artifacts.

We did the same spectral analysis to macro-pixels out of the fan structure, only the orbital artifacts and/or other random peaks due to noise or unknown sources were found. It suggests that the peaks possibly of solar origin are only localised to the diffuse structure. We enlarged the macro-pixel size from $3 \times 3$ pixels to $20 \times 20$ pixels, the corresponding power spectrum is shown in Fig. 4c. Since the orbital artifact exist in all pixels, it is seen that the rest of the peaks are smoothed out and buried relatively in the enhanced orbital harmonics. In order to identify the disappearing peaks and distinguish signals of solar origin from the noise within the data and artifacts due to spectral leakage of the orbital harmonics, we also performed filtering to the signal, as specified in Sect. 2.4. The spectrum (see Fig. 4e) after filtering out the orbital harmonics, shows significant peaks. The result is confirmed by the $195 \AA$ data, displayed in Fig. 4b,d and f. The results are summarised in Table 1 . 
D. Yuan et al.: Leakage of long-period oscillations from the chromosphereto the corona

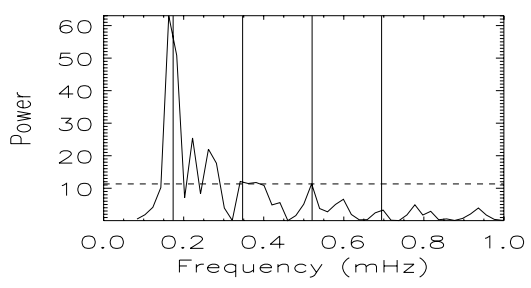

(a)

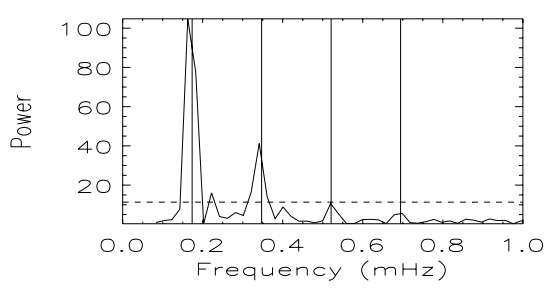

(c)

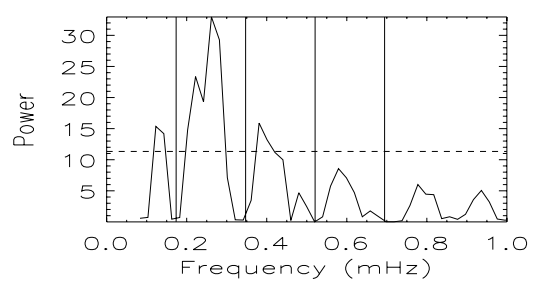

(e)

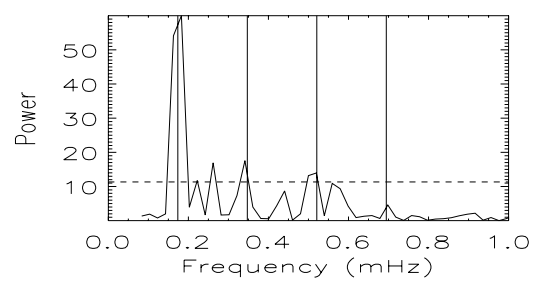

(b)

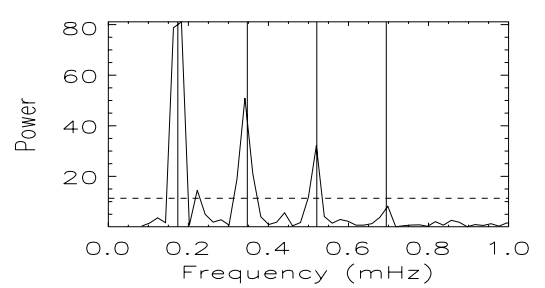

(d)

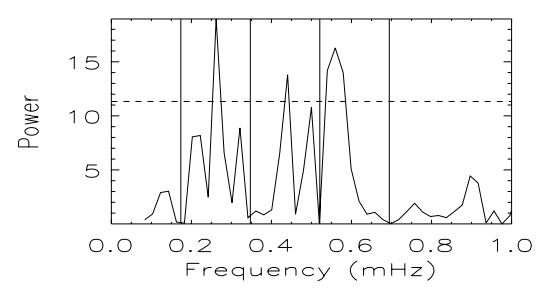

(f)

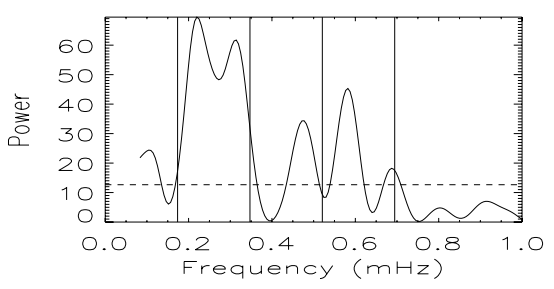

(g)

Fig. 4. a), b) Power spectra of the time series (1998-07-03 00:47-09:00 UT) of a macro-pixel ( $3 \times 3$ pixels) situated along the mid-way of the slit (Fig. 1b in EUV $171 \AA$ (left) and $195 \AA$ (right). c), d) The corresponding spectra of an enlarged macro-pixel $(20 \times 20$ pixels). e), f) The power spectra after filtering out the orbital frequencies. g) The power spectrum of NoRH 17 GHz radio emission, (1998-07-03 02:55-06:30 UT). The vertical dot-dot-dashed lines are the orbital frequency and its higher harmonics. A horizontal dashed line indicates the $99 \%$ confidence level in all the panels (see Sect. 2.4). The detected peaks in all the bandpasses are summarised in Table 1.

Outside the orbital periodicity and its higher harmonics, we detected significant spectral peaks at $0.123,0.222,0.262,0.302$, $0.382,0.421$ and $0.579 \mathrm{mHz}$ in the TRACE EUV $171 \AA$ emission from a fan-like coronal structure in the active region. These peaks are detected with FAP $(p<0.01)$ in both Horne \& Baliunas (1986) and Fisher randomisation test (see Fig. 5 and Table 1). In the EUV $195 \AA$ data, we only detected 0.262 and $0.559 \mathrm{mHz}$ with $F A P<0.01$ in both two significance tests. In the Horne \& Baliunas (1986) test, the peaks at 0.202 and $0.320 \mathrm{mHz}$ were found to have $F A P>0.01$, while in the Fisher randomisation test, the former peak was found significant $(p<0.01)$ and the latter was detected with poor confidence $(p=0.20)$. The FAPs of 0.440 and $0.500 \mathrm{mHz}$ are both $<0.01$ in Horne \& Baliunas (1986) test and estimated at $p=0.018$ and $p=0.025$ in randomisation test respectively (see Fig. 6 and Table 1).

Similar analysis was performed to the $17 \mathrm{GHz}$ radio intensity data obtained with NoRH, which is ground-based and free of the orbital effects. The available time series was 1998-07-03 02:5506:30 UT. The power spectrum indicates the existence of longperiod oscillations (Fig. $4 \mathrm{~g}$ ). We found spectral peaks in 0.220 , $0.314,0.467$ and $0.582 \mathrm{mHz}$ with very good confidence $(p<$ 0.01) in both Horne \& Baliunas (1986) and Fisher randomisation tests (see Fig. 7 and Table 1).

\section{Conclusions}

In this study, we selected time sequences from a five day sample of TRACE observation data over AR8253 to study the long period coronal oscillations. The spacecraft orbital period creates a strong artificial periodicity in the light curves. We found that the TRACE orbital frequency $(0.17 \mathrm{mHz})$ and its higher harmonics $(0.35,0.52$ and $0.69 \mathrm{mHz})$ are pronounced in the EUV intensity images, because of its nonlinear dependence on the CCD temperature variation. A similar artifact can be present in the data obtained with other sun-synchronous space missions, e.g. Yohkoh, Hinode and CORONAS-Photon. 

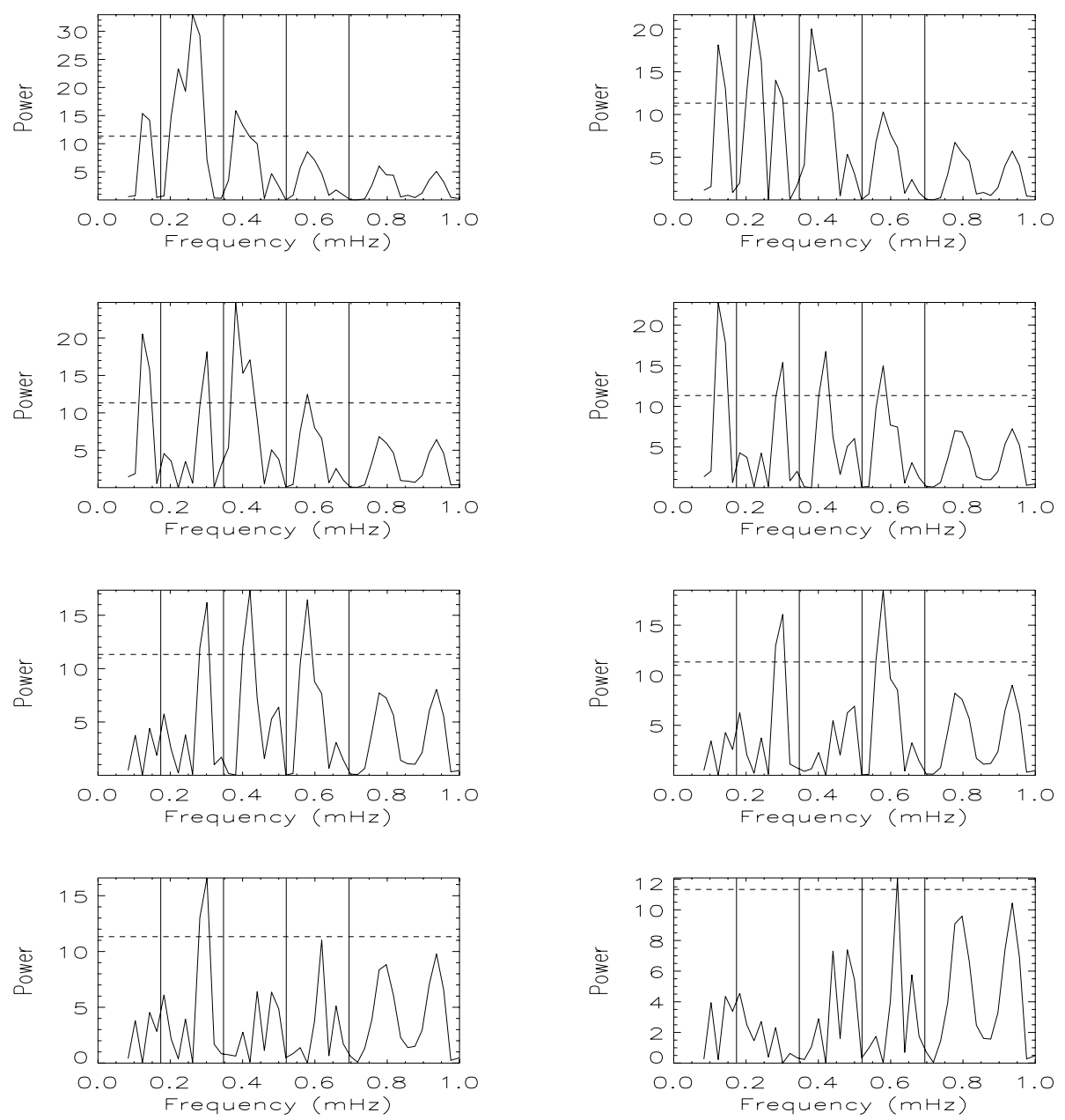

Fig. 5. Lomb-Scargle periodograms of $171 \AA$ data shown after iteratively subtracting the highest peak in the spectrum with power above $1 \%$ of $F A P$ (dashed line). The iteration is displayed in order from left to right, top to bottom.
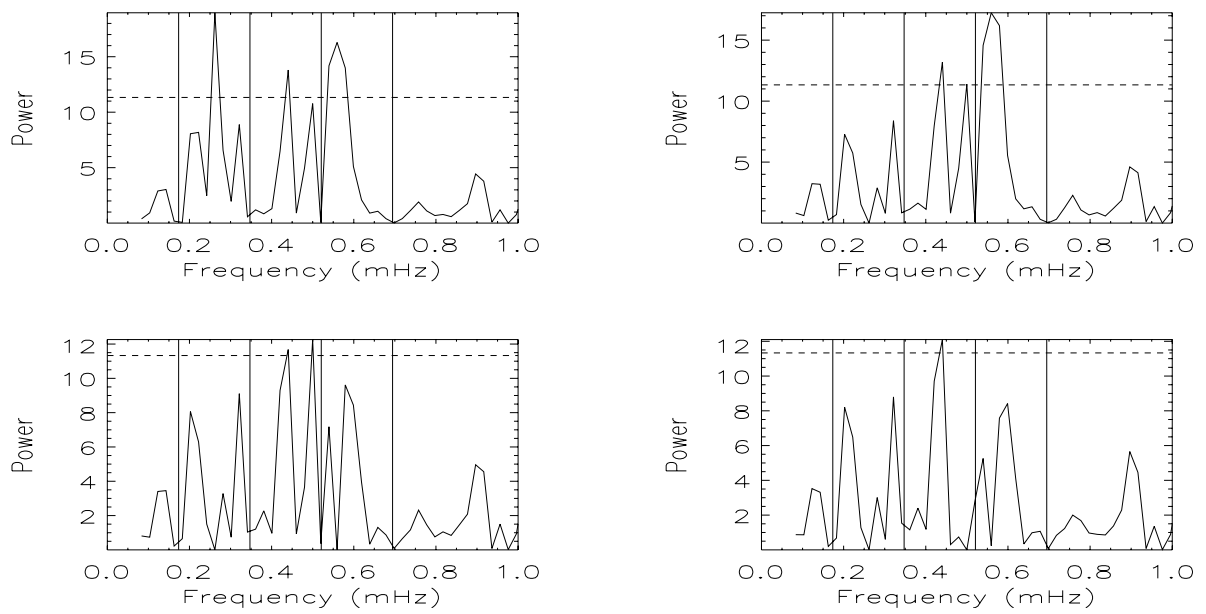

Fig. 6. The same analysis to $195 \AA$ data as in Fig. 5. The iteration stops earlier than those for $171 \AA$ data, but we note peaks with power below $99 \%$ confidence level, near 0.202 and $0.320 \mathrm{mHz}$, that may be of relevance.

We note that $0.221,0.312,0.573 \mathrm{mHz}$ (average values, see Table 1) oscillations are present in both EUV emissions in the corona and radio signal in the chromosphere, they are found with with good confidence in both Horne \& Baliunas (1986) and Fisher randomisation test at both levels of the solar atmosphere. We conclude that the $0.221,0.312,0.573 \mathrm{mHz}$ spectral peaks are of solar origin, and are present in the chromosphere and in the corona. Since the diffuse structure in the corona is the magnetic field extension of the sunspot, propagating EUV disturbance (slow magnetoacoustic wave, see introduction in Sect. 1) was seen persistent for days in the datasets, they were found to leak from the sunspot (Shibasaki 2001; Sych et al. 2009), it strongly implies as well the connectivity of the long period oscillations in the chromosphere and corona.

The nature of the long-period oscillations has not been understood yet. It can have several interpretations. For example, 
D. Yuan et al.: Leakage of long-period oscillations from the chromosphereto the corona
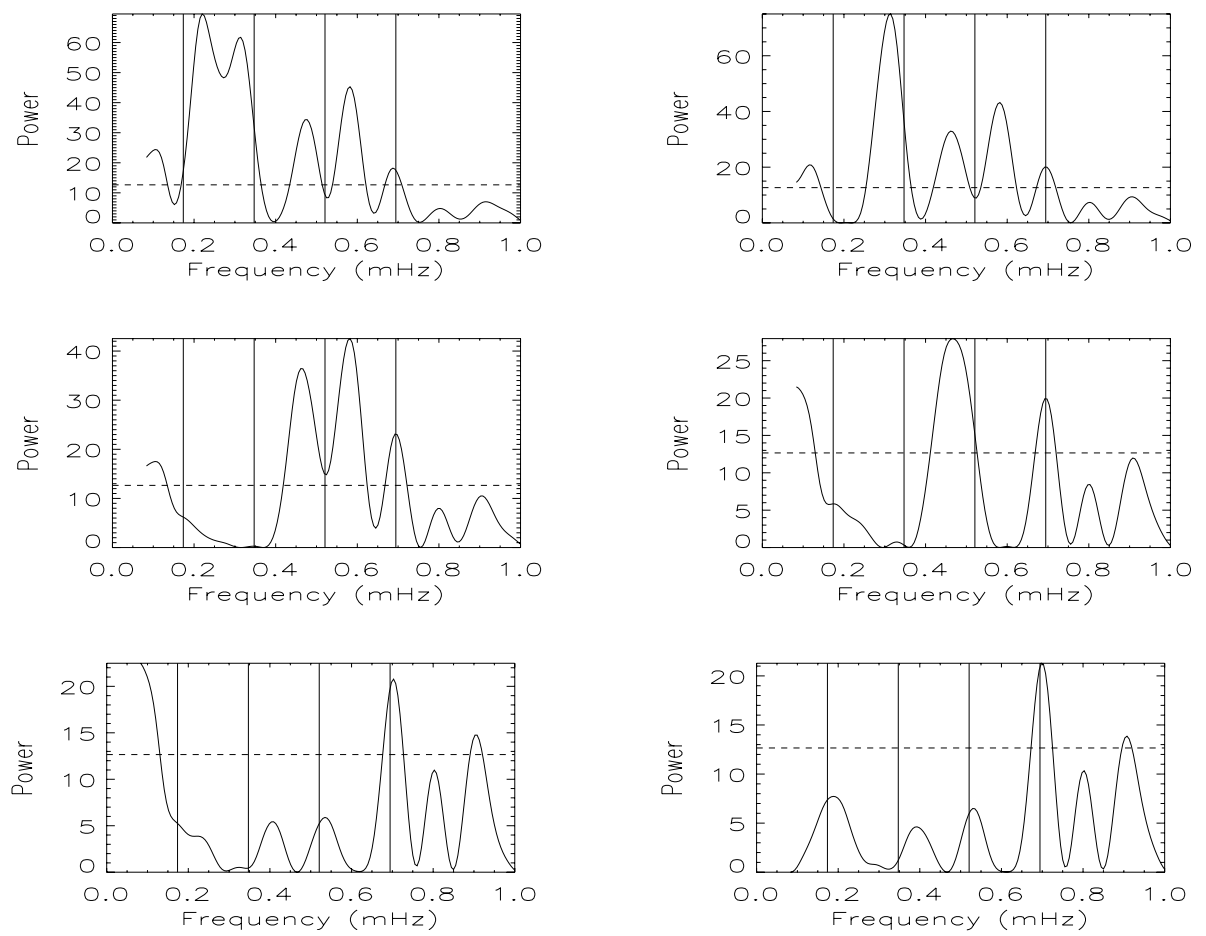

Fig. 7. The same analysis to NoRH $17 \mathrm{GHz}$ data as in Fig. 5 .

Table 1. Summary of the detected peaks and significance tests.

\begin{tabular}{cccccccc}
\hline \hline \multicolumn{2}{c}{ Bandpass } & & $0.17 \sim 0.35 \mathrm{mHz}$ & \multicolumn{3}{c}{$0.35 \sim 0.52 \mathrm{mHz}$} & $0.52 \sim 0.69 \mathrm{mHz}$ \\
\hline \multirow{2}{*}{ TRACE 171 $\AA$} & $f(\mathrm{mHz})$ & $0.222 \pm 0.008$ & $0.262 \pm 0.008$ & $0.302 \pm 0.008$ & $0.382 \pm 0.008$ & $0.421 \pm 0.008$ & $0.579 \pm 0.008$ \\
& $P(\mathrm{~min})$ & $75 \pm 2.7$ & $64 \pm 1.9$ & $55 \pm 1.4$ & $44 \pm 0.9$ & $39 \pm 0.8$ & $29 \pm 0.4$ \\
& $p$-value & $<0.01$ & $<0.01$ & $<0.01$ & $<0.01$ & $<0.01$ & $<0.01$ \\
TRACE 195 $\AA$ & $f(\mathrm{mHz})$ & {$[0.202 \pm 0.008]$} & $0.262 \pm 0.008$ & {$[0.320 \pm 0.008]$} & $0.440 \pm 0.009$ & $0.500 \pm 0.008$ & $0.559 \pm 0.009$ \\
& $P(\mathrm{~min})$ & {$[83 \pm 3.3]$} & $64 \pm 1.9$ & {$[52 \pm 1.3]$} & $37 \pm 0.8$ & $33 \pm 0.5$ & $30 \pm 0.5$ \\
& $p$-value & $<0.01$ & $<0.01$ & 0.20 & 0.018 & 0.025 & $<0.01$ \\
NoRH 17 GHz & $f(\mathrm{mHz})$ & $0.220 \pm 0.020$ & $\ldots$ & $0.314 \pm 0.019$ & $0.467 \pm 0.020$ & $\ldots$ & $0.582 \pm 0.019$ \\
& $P(\mathrm{~min})$ & $75 \pm 6.9$ & $\ldots$ & $53 \pm 3.2$ & $36 \pm 1.5$ & $\ldots$ & $29 \pm 0.93$ \\
& $p$-value & $<0.01$ & $\ldots$ & $<0.01$ & $<0.01$ & $\ldots$ & $<0.01$ \\
\hline Average frequency $(\mathrm{mHz})$ & $0.221 \pm 0.020$ & $\ldots$ & $0.312 \pm 0.020$ & $\ldots$ & $\ldots$ & $0.573 \pm 0.020$ \\
\hline
\end{tabular}

Notes. All listed frequencies and corresponding periods have $F A P<0.01$ according to the Horne \& Baliunas (1986) test, except for the values indicated between square brackets (found in the $195 \AA$ time series and of potential relevance given their closeness to significant values found in other bandpasses). The $p$-value indicates the false alarm probability from the Fisher randomisation test.

the $0.312 \mathrm{mHz}$ oscillation was also well detected in $17 \mathrm{GHz}$ radio emission in sunspot oscillations (Chorley et al. 2010, 2011). The $0.221 \mathrm{mHz}$ oscillations are very close to the $l=2, n=-3$ or $l=3, n=-5 g$-mode (note: the same $2 l+s$ combinations pronounce the same frequency, see Turck-Chièze et al. 2004), while the $0.583 \mathrm{mHz}$ could be associated with the $l=1 \mathrm{~g}$-mode (see García et al. 2007). The slight discrepancy can be attributed to observational uncertainties, unresolved multiplet splitting at azimuthal order $m$ due to solar interior rotation or other dynamical processes. In this case, one can speculate that the energy of $g$-modes, which cannot reach the solar surface, can be channelled by the magnetic field from the deeper layers of the solar interior, especially the less efficiently trapped low- $l$ modes, and hence be seen in the regions of the magnetic concentrations such as the sunspots. The variations of the parameters of the plasma surrounding a magnetic flux tube in the convection zone by a $g$-mode could excite trapped MHD waves propagating along the tube to the solar surface. Thus, the magnetic flux tubes forming the sunspot could act as a waveguide with little attenuation of the $g$-mode signal. However, rigorous analysis of this mechanism remains to be performed. Another possibility is the global oscillations of the sunspot, proposed in terms of the shallow sunspot model (Solov'ev \& Kirichek 2008).

Another puzzle is the presence of the long-period oscillations in the corona, found in this study. Waves with the periods much longer than the acoustic cut-off period (normally 200-300 s in the chromospheric plasma) (Bel \& Leroy 1977) are believed to be constrained to the chromosphere. One possibility is that the wave is indeed evanescent above the chromosphere, but its penetration depth is sufficiently large to be detected in the corona. Another option could be some kind of wave-guiding by the magnetic field, while its nature needs to be revealed. However, it could not be ruled out that the detected long-period oscillations in the chromosphere and in the corona have the same period by pure coincidence.

In any case, the detection of long-period oscillations in the lower corona is a promising signal to help the understanding of the magnetic connectivity of the different layers of the Sun and beyond. 
Acknowledgements. D.Y. acknowledges the MPAGS funding for studentship. N.C. is supported by EPSRC studentship. C.F. acknowledges financial support from the UK Science and Technology Facilities Council (STFC) on the CFSA Rolling Grant. We thank the anonymous referee for helpful comments. We acknowledge the TRACE team for providing data. NoRH is operated by the Nobeyama Solar Radio Observatory.

\section{References}

Appourchaux, T., Belkacem, K., Broomhall, A. M., et al. 2010, A\&ARv, 18, 197 Aschwanden, M. J., Nightingale, R. W., Tarbell, T. D., \& Wolfson, C. J. 2000, ApJ, 535, 1027

Bel, N., \& Leroy, B. 1977, A\&A, 55, 239

Berghmans, D., \& Clette, F. 1999, Sol. Phys., 186, 207

Botha, G. J. J., Arber, T. D., Nakariakov, V. M., \& Zhugzhda, Y. D. 2011, ApJ, 728,84

Chorley, N., Hnat, B., Nakariakov, V. M., Inglis, A. R., \& Bakunina, I. A. 2010, A\&A, 513, A27

Chorley, N., Foullon, C., Hnat, B., Nakariakov, V. M., \& Shibasaki, K. 2011, A\&A, 529, A123

De Moortel, I., Ireland, J., \& Walsh, R. W. 2000, A\&A, 355, L23

De Moortel, I., Hood, A. W., Ireland, J., \& Walsh, R. W. 2002a, Sol. Phys., 209, 89

De Moortel, I., Ireland, J., Walsh, R. W., \& Hood, A. W. 2002b, Sol. Phys., 209, 61

De Pontieu, B., Erdélyi, R., \& De Moortel, I. 2005, ApJ, 624, L61

Deforest, C. E., \& Gurman, J. B. 1998, ApJ, 501, L217

Dhillon, V. S., Privett, G. J., \& Duffey, K. P. 2001, PERIOD, A Time-Series Analysis Package Version 5.0 User's Manual

Efremov, V. I., Parfinenko, L. D., \& Soloviev, A. A. 2009, Cosmic Research, 47, 279

Ferraz-Mello, S. 1981, AJ, 86, 619

Foullon, C., Verwichte, E., \& Nakariakov, V. M. 2004, A\&A, 427, L5

Foullon, C., Verwichte, E., \& Nakariakov, V. M. 2009, ApJ, 700, 1658
García, R. A., Turck-Chièze, S., Jiménez-Reyes, S. J., et al. 2007, Science, 316, 1591

Gelfreikh, G. B., Nagovitsyn, Y. A., \& Nagovitsyna, E. Y. 2006, PASJ, 58, 29

Gounder, M. K., Shitan, M., \& Imon, R. A. 2007, in Festschrift in honor of Distinguished Professor Mir Masoom Ali On the occasion of his retirement, 213

Handy, B. N., Acton, L. W., Kankelborg, C. C., et al. 1999, Sol. Phys., 187, 229 Hernandez, G. 1999, J. Geophys. Res., 104, 10355

Hershaw, J., Foullon, C., Nakariakov, V. M., \& Verwichte, E. 2011, A\&A, 531, A53

Horne, J. H., \& Baliunas, S. L. 1986, ApJ, 302, 757

Inglis, A. R., \& Nakariakov, V. M. 2009, A\&A, 493, 259

Kepko, L., \& Spence, H. E. 2003, J. Geophys. Res. (Space Physics), 108, 1257

King, D. B., Nakariakov, V. M., Deluca, E. E., Golub, L., \& McClements, K. G. 2003, A\&A, 404, L1

Linnell Nemec, A. F., \& Nemec, J. M. 1985, AJ, 90, 2317

Marsh, M. S., Walsh, R. W., \& Plunkett, S. 2009, ApJ, 697, 1674

McIntosh, S. W., de Pontieu, B., \& Tomczyk, S. 2008, Sol. Phys., 252, 321

Nagovitsyna, E. Y., \& Nagovitsyn, Y. A. 2001, Astron. Lett., 27, 118

Nagovitsyna, E. Y., \& Nagovitsyn, Y. A. 2002, Astron. Lett., 28, 121

Nakariakov, V. M., Verwichte, E., Berghmans, D., \& Robbrecht, E. 2000, A\&A, 362,1151

Ofman, L., Romoli, M., Poletto, G., Noci, G., \& Kohl, J. L. 2000, ApJ, 529, 592

O'Shea, E., Banerjee, D., Doyle, J. G., Fleck, B., \& Murtagh, F. 2001, A\&A, 368,1095

Scargle, J. D. 1982, ApJ, 263, 835

Shibasaki, K. 2001, ApJ, 550, 1113

Solov'ev, A. A., \& Kirichek, E. A. 2008, Astrophys. Bull., 63, 169

Sych, R., Nakariakov, V. M., Karlicky, M., \& Anfinogentov, S. 2009, A\&A, 505, 791

Thomson, D. J., Maclennan, C. G., \& Lanzerotti, L. J. 1995, Nature, 376, 139

Tripathi, D., Isobe, H., \& Jain, R. 2009, Space Sci. Rev., 149, 283

Turck-Chièze, S., García, R. A., Couvidat, S., et al. 2004, ApJ, 604, 455

Verwichte, E., Marsh, M., Foullon, C., et al. 2010, ApJ, 724, L194

Wang, T. J., Ofman, L., Davila, J. M., \& Mariska, J. T. 2009, A\&A, 503, L25 